\title{
Influence of fluvoxamine on plasma interleukin-6 or clinical improvement in patients with major depressive disorder
}

This article was published in the following Dove Press journal:

Neuropsychiatric Disease and Treatment

14 February 2017

Number of times this article has been viewed

\section{Reiji Yoshimura \\ Asuka Katsuki \\ Kiyokazu Atake \\ Hikaru Hori \\ Ryohei Igata \\ Yuki Konishi}

Department of Psychiatry, University of Occupational and Environmental Health, Yahatanishi-ku, Kitakyushu,

Fukuoka, Japan
Correspondence: Reiji Yoshimura Department of Psychiatry, University of Occupational and Environmental Health, I-I Iseigaoka, Yahatanishi-ku, Kitakyushu, Fukuoka 8078555, Japan

Tel +8| 93 69| 7253

Fax $+8 I 936924894$

Email yoshi621@med.uoeh-u.ac.jp
Objectives: The etiology of depression remains unknown. There is, however, a growing body of evidence that cytokines are involved in the pathophysiology of depression. The aim of this study is to investigate the effects of fluvoxamine on plasma interleukin-6 (IL-6) levels and on clinical improvement of the depressive state.

Subjects and methods: Thirty patients who met the DSM-IV criteria for major depressive disorder (MDD) were enrolled in the study. Thirteen were male and 17 were female, and their ages ranged from 26 to 70 years (mean \pm standard deviation $45.0 \pm 14.2$ ). The patients were treated with fluvoxamine for 8 weeks. The dosages of fluvoxamine varied among the patients and, based on ethical considerations, were not fixed.

Results: The fluvoxamine doses were positively related to plasma fluvoxamine levels $(r=0.8798$, $P<0.001)$. A significant correlation was observed between the patients' plasma IL-6 levels and their 17-item Hamilton Rating Scale for Depression (HAMD17) scores $(r=0.4555, P=0.0010)$. A positive correlation was found between the delta plasma IL-6 (week 0-week 8) and the delta HAMD17 (week 0-week 8) ( $r=0.5226, P=0.002)$.

Conclusion: Effect of fluvoxamine on IL-6 is partially associated with its clinical efficacy for MDD.

Keywords: fluvoxamine, interleukin, Hamilton Rating Scale for Depression, major depressive disorder

\section{Introduction}

Selective serotonin reuptake inhibitors (SSRIs) are the first-line treatment for major depressive disorder (MDD). ${ }^{1}$ SSRIs work on the serotonin transporter (SET), and Coleman et $\mathrm{al}^{2}$ recently used X-ray crystallography to determine two structures of the SET that were bound to an SSRI. Serotonin might play an important role in the pathophysiology of MDD. However, the etiology of depression remains unknown. There is a growing body of evidence that cytokines are involved in the pathophysiology of depression. ${ }^{3,4}$ Activated microglia exhibit increased production of inflammatory cytokines (eg, interleukin (IL)-1 $\beta$, tumor necrosis factor-alpha (TNF- $\alpha$ ), and IL-6). ${ }^{3}$ According to the recent meta-analysis, levels of two cytokines (IL-6 and TNF- $\alpha$ ), one soluble cytokine receptor and one cytokine receptor antagonist were significantly increased in acutely ill patients with MDD compared with controls. ${ }^{5}$ Another former meta-analysis demonstrated that a significantly higher concentrations of the proinflammatory cytokines TNF- $\alpha$ and IL-6 in depressed subjects compared with control subjects. ${ }^{6}$ Antidepressant treatment also reduced levels of IL-1 $\beta$ and possibly those of IL-6. ${ }^{7}$ It is well-known that noradrenaline stimulates the release of cytokines from 
microglia and macrophages, and the effect of noradrenaline and cytokines is regulated by adrenoceptors. ${ }^{3}$ We reported that the plasma levels of IL- 6 are raised during depressive periods. ${ }^{8,9}$ According to a meta-analysis 7, treatment with antidepressants reduced the serum IL-6 levels in patients with MDD. In other words, plasma IL-6 is a state marker of major depressive episodes. To the best of our knowledge, there are no reports of investigations into the responses to fluvoxamine, an SSRI, and plasma IL-6 levels in patients with MDD. Thus, this study examined the responses to fluvoxamine and plasma IL-6 levels in MDD patients. An activation of the immune system has repeatedly been described in MDD, and it has been suggested that the cytokines play a key role in this immune activation. ${ }^{3,4}$ Within the brain, cytokines exert various effects on neurotransmitters - especially the noradrenaline systems, which are thought to play a pivotal role in psychiatric disorders. ${ }^{3}$ IL-6 receptors are widely distributed in the brain, especially in the hippocampus and hypothalamus. ${ }^{10}$ Maes ${ }^{11}$ demonstrated that the plasma concentrations of IL-6 were significantly higher in MDD subjects than in healthy controls. This finding has been confirmed by a number of subsequent meta-analyses. ${ }^{12}$ The above findings suggested that IL-6 might play a role in the pathogenesis of MDD. From the above findings, it may be surmised that IL-6 may play a role in the biology or somatic consequences of MDD. Fluvoxamine is an SSRI that is widely used in the treatment of depression and other psychiatric disorders including anxiety disorders, and it has been suggested to have early effects when used as an antidepressant drug. ${ }^{13,14}$ In addition, the results of a recent meta-analysis also suggest that treatment with fluvoxamine leads to symptomatic improvements in patients with MDD by the end of the first week of use. ${ }^{15}$

The aim of this study is to investigate the effects of fluvoxamine on plasma IL-6 levels, especially focusing on the dose or plasma level of fluvoxamine and the clinical improvement of the depressive state.

\section{Subjects and methods}

Thirty patients who met the DSM-IV criteria (American Psychiatric Association, 1994) for MDD without other Axis I or Axis II disorders were enrolled in the study. Thirteen were male and 17 were female, and their ages ranged from 26 to 70 years (mean \pm standard deviation $=45.0 \pm 14.2$ ). All patients were physically healthy and free of current alcohol and/or drug abuse. The patients were treated with fluvoxamine for 8 weeks. The dosages of fluvoxamine varied among the patients and, based on ethical considerations, were not fixed.
Depressive symptoms were evaluated using the 17-item Hamilton Rating Scale for Depression (HAMD17). ${ }^{16}$ The patients' baseline blood samples drawn into heparinized tubes were obtained before breakfast at $08.00-10.00$ prior to the initiation of SSRI treatment. None of the patients took any medications for at least a month before the blood samplings. The plasma samples were quickly separated in a centrifuge and stored at $-80^{\circ} \mathrm{C}$ until they were assayed.

The plasma levels of IL- 6 were measured with our quantitative sandwich enzyme assay technique, which we previously described using a Quantikine ${ }^{\circledR}$ HS High Sensitivity Immunoassay kit (R\&D Systems, Minneapolis, MN, USA). ${ }^{17}$ The standard curve was linear from 0.1 to $10 \mathrm{pg} / \mathrm{mL}$, and the detection limit was $0.1 \mathrm{pg} / \mathrm{mL}$. The intra- and interassay coefficients of variation were $7 \%$ and $9 \%$, respectively. The recovery rates of the exogenously added IL-6 in the measured plasma samples were $>95 \%$.

The protocol of this study was approved by the Ethics Committee of the University of Occupational and Environmental Health, Kitakyushu, Japan. Written informed consent was obtained from all subjects. The plasma fluvoxamine level was measured using high performance liquid chromatography according to the method we previously described. ${ }^{18}$ In brief, $1 \mathrm{~mL}$ of plasma alkalized with $500 \mu \mathrm{L}$ of $2 \mathrm{M}$ sodium hydrogen carbonate was extracted by hexane $(10 \mathrm{~mL})$ after the addition of the internal standard (clomipramine). Shaken horizontally for $20 \mathrm{~min}$ and then centrifuged at $2,000 \times g$ for $10 \mathrm{~min}$, the upper organic layer was removed and dried under $\mathrm{N}_{2}$. After being dissolved in $200 \mu \mathrm{L}$ of mobile phase, a $50 \mu \mathrm{L}$ aliquot of the final preparation was subjected to high performance liquid chromatography. All experiments were performed in triplicate.

\section{Statistical analysis}

Since the data were normal distributed using KolmogorovSmirnov test. A paired $t$-test was used to compare the plasma IL-6 levels and the HAMD17 scores between at week 0 and at week 8. Relationships between two variables were examined using Pearson's correlation coefficients. The level of significance for all analyses was set at $P<0.05$.

\section{Results}

1) The demographics of each group are given in Table 1; 2) the fluvoxamine dose was positively related to the plasma fluvoxamine level (Pearson's correlation coefficients; $r=0.8798, P<0.001$; Figure 1); 3 ) plasma IL-6 levels decreased from week $0(134 \pm 53 \mathrm{pg} / \mathrm{mL})$ to week 8 
Table I Demographics of participants

\begin{tabular}{ll}
\hline$M(F)$ & $13(17)$ \\
Age (years) & $45.0 \pm 14.2$ \\
First/recurrent & $12(18)$ \\
Duration of illness & $9.4 \pm 3.6$ (weeks) \\
Fluvoxamine dose (max) & $111.2 \pm 37.4$ (mg/day) \\
HAMDI7 (at week 0) & $24.1 \pm 3.1$ \\
HAMDI7 (at week 8) & $12.2 \pm 2.8$ \\
\hline
\end{tabular}

Abbreviations: HAMDI7, 17-item Hamilton Rating Scale for Depression; max maximum; $M$, male; $F$, female.

(106 $\pm 34 \mathrm{pg} / \mathrm{mL}$; paired Student's $t$-test, $P=0.0183) ; 4$ ) the HAMD17 scores decreased week $0(24.4 \pm 3.1)$ to week 8 (12.2 \pm 2.8 ; paired $t$-test, $P=0.0001) ; 5)$ a significant correlation was observed between the patients' plasma IL-6 levels and their HAMD17 scores (Pearson's correlation coefficients; $r=0.4555, P=0.0010$; Figure 2$)$; 6 ) a positive correlation was found between the delta plasma IL-6 (week 0-week 8) and the delta HAMD17 (week 0-week 8) (Pearson's correlation coefficients; $r=0.5226, P=0.002$; Figure 3 ); 7) no correlation was found between plasma fluvoxamine level at week 8 and the delta HAMD17 (Pearson's correlation coefficients; $r=1.3045, P=0.2020$; Figure 4); 8) no correlation was observed between delta plasma IL-6 (week 0-week 8) and plasma fluvoxamine level at week 8 (Pearson's correlation coefficients; $r=0.2982, P=0.7676$; Figure 5).

\section{Discussion}

The main finding of this study was that a positive correlation was observed between the delta plasma IL- 6 and the delta HAMD17 in MDD patients treated with fluvoxamine. This indicates that fluvoxamine an association exists between the decrease in plasma IL- 6 and the clinical improvement of depressive symptoms. We also demonstrated that the plasma IL-6 level was associated with the severity of depressive symptoms evaluated by HAMD17. Recently, we reported that

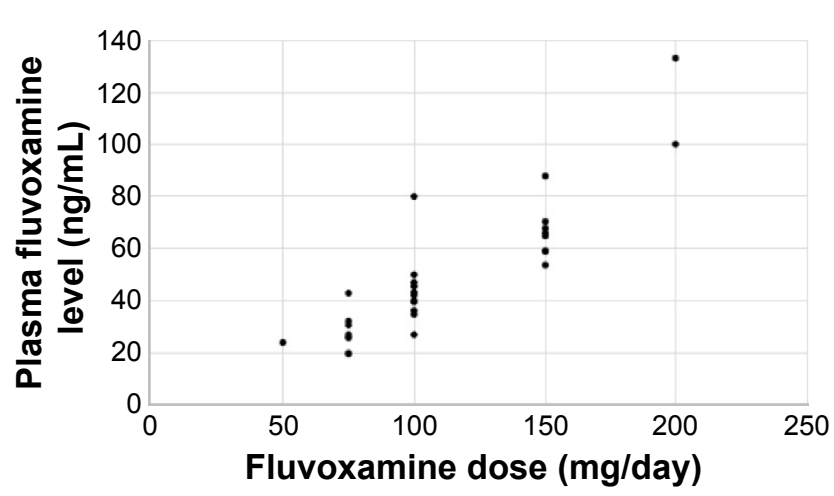

Figure I Fluvoxamine dose and plasma fluvoxamine concentration. Notes: $r=0.8798, P<0.00 \mathrm{I}$

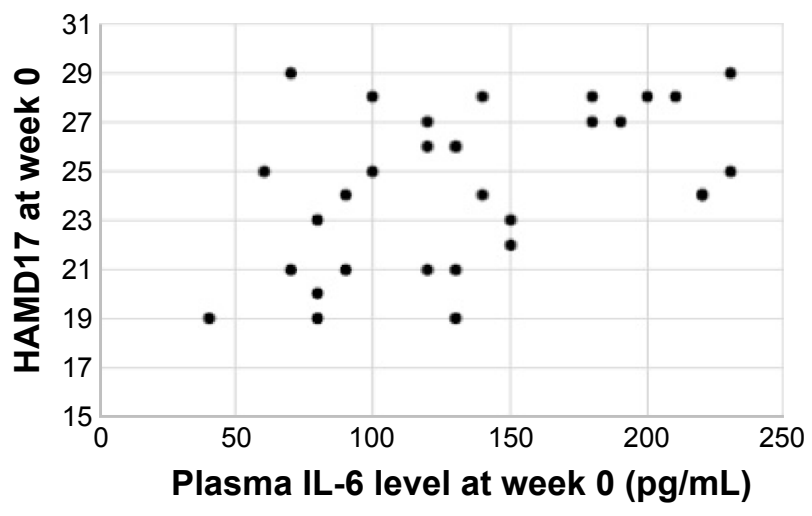

Figure 2 Baseline (week 0) HAMDI7 and baseline (week 0) plasma IL-6 concentration.

Notes: $r=0.4555, P=0.0010$

Abbreviations: HAMDI7, 17-item Hamilton Rating Scale for Depression; IL-6, interleukin-6.

the baseline plasma IL-6 levels were significantly higher in responders to paroxetine or sertraline than in nonresponders. In that study, a significant positive correlation was observed between the patients' HAMD17 scores and the baseline plasma IL-6 levels 5. We reconfirmed in this study that plasma IL-6 reflects the severity of major depressive episodes. Taking all of these findings into account, it is possible that plasma IL-6 is a biological state marker for MDD.

IL-6 has been proposed to be involved in the pathology of MDD. ${ }^{18}$ In particular, it was suggested that IL-6 is involved in multiple physiological systems, including the hypothalamic-pituitary-adrenal axis, corticotrophinreleasing hormone activity at limbic sites, noradrenaline utilization, the release of oxidative stress, the apoptotic pathways, and kinase signaling, ${ }^{19-22}$ all of which have very close relationships to the pathophysiology of MDD. Li et $\mathrm{al}^{23}$ recently showed that noradrenaline significantly increased the mRNA and protein expression of IL-6 in

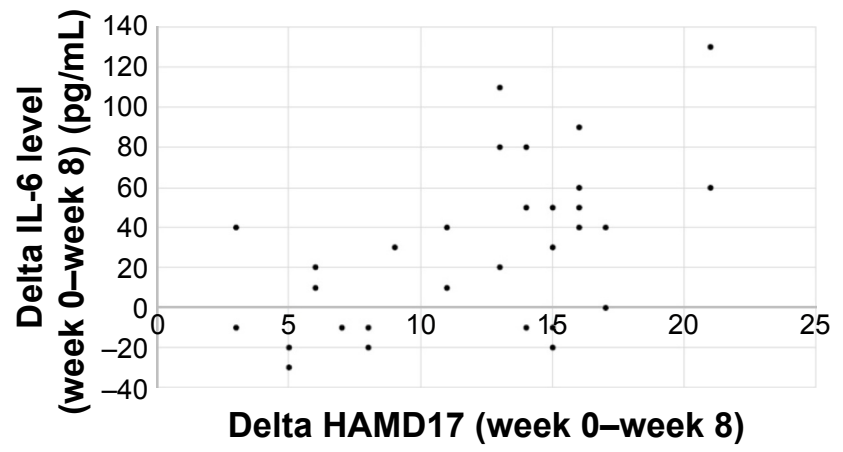

Figure 3 The change in plasma IL-6 concentration and the change in HAMDI7. Notes: $r=0.5226, P=0.002$.

Abbreviations: HAMDI7, 17-item Hamilton Rating Scale for Depression; IL-6, interleukin-6. 


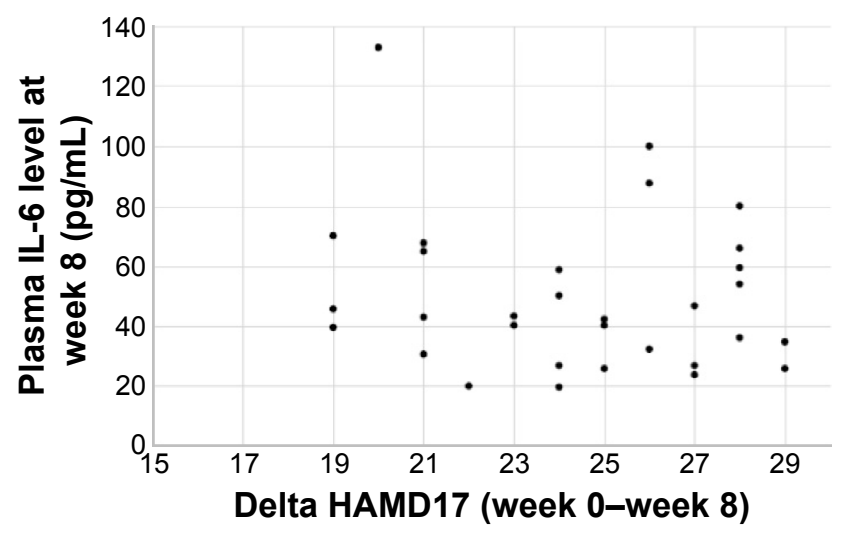

Figure 4 The change in HAMDI7 and plasma IL-6 concentration at week 8. Notes: $r=1.3045, P=0.2020$.

Abbreviations: HAMDI7, 17-item Hamilton Rating Scale for Depression; IL-6, interleukin-6.

U937 macrophages in time- and concentration-dependent manners. The $\beta$-adrenoreceptor inhibitor propranolol blocked noradrenaline-induced IL-6 expression in the mRNA and protein levels in U937 macrophages, which hypernoradrenergic activity promotes IL-6 production. Actually, we reported that plasma 3-methoxy-4-hydroxyphenylglycol, a major metabolite of noradrenaline, was higher in the subgroup of MDD patients than in healthy control subjects, and also demonstrated that fluvoxamine decreased the plasma 3-methoxy-4-hydroxyphenylglycol level, which was associated with the clinical improvement of depressive symptoms. ${ }^{24}$ Li et $\mathrm{al}^{23}$ reported that the MHPG levels were elevated with anxiety, agitation, and insomnia, and the authors clonidine, an alpha2-adrenoceptor agonist, was effective for certain depressive symptoms. Therefore, suppressing noradrenaline activity enhanced the serotonergic functions by fluvoxamine leads to weaken IL-6 production in MDD patients. In other words, fluvoxamine increases brain serotonin, and increased

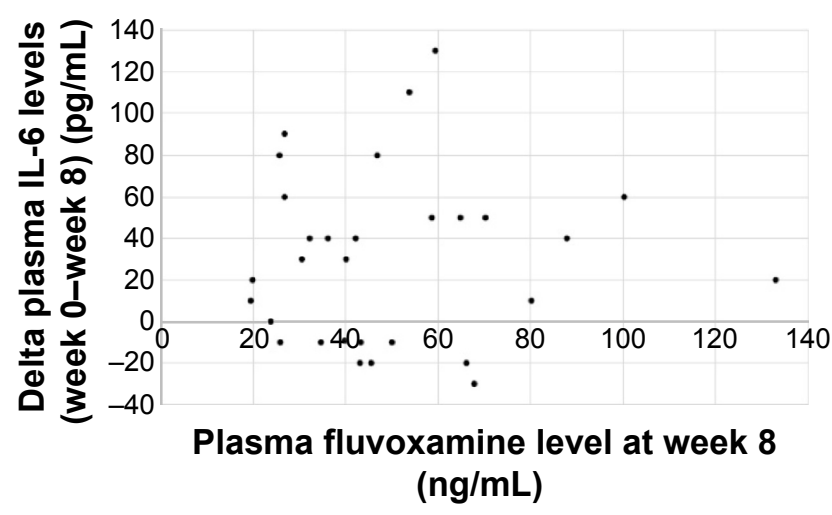

Figure 5 The change in plasma IL-6 concentration and plasma fluvoxamine concentration at week 8 .

Notes: $r=0.2982, P=0.7676$

Abbreviation: IL-6, interleukin-6. serotonin stimulates the alpha2-adrenenoceptor, which reduces noradrenergic activity. ${ }^{25} \mathrm{In}$ short, an interaction exists between noradrenergic neurons and serotonergic neurons.

Microglia are the primary central reservoirs of proinflammatory cytokines, and they act as antigen-presenting cells in the brain. ${ }^{26}$ Although it is uncertain whether peripheral IL-6 levels represent the levels found in the brain, evidence suggests that IL- 6 crosses the blood-brain barrier, and the peripheral level might represent an overflow from the central nervous system and may be a marker of an inflammatory process in the brain. ${ }^{27}$

A linear relationship was found between the dose and plasma concentrations of fluvoxamine. No association was found, however, between plasma fluvoxamine level and the delta HAMD17. These results suggest that the dose and plasma levels of fluvoxamine independently influenced the clinical improvement of depressive symptoms. Pharmacodynamic factors and pharmacokinetic factors are both important for the clinical efficacy of fluvoxamine in MDD. Since placebo control group was not prepared, the possibility remains that reduction of plasma IL-6 levels might be related to recovery itself, and do not necessarily have to be a pharmacological effect of fluvoxamine.

\section{Limitations}

One limitation of this study was that the sample size was small and heterogeneous, not reference group, not placebo group, which might be associated with a type I or type II error. The fact that the dosage of fluvoxamine was not fixed is another problem. Thus, further research using larger sample with reference group, a different antidepressant, is needed to test the preliminary results achieved in this study.

\section{Conclusion}

The effect of fluvoxamine on IL-6 is related to its clinical efficacy of fluvoxamine for MDD. We should, however, be cautious for interpreting the preliminary results.

\section{Acknowledgment}

The study was supported by a Health and Labor Research grant (\#1401010101).

\section{Disclosure}

The authors report no conflicts of interest in this work.

\section{References}

1. Jakubovski E, Varigonda AL, Freemantle N, Taylor MJ, Bloch MH. Systematic review and meta-analysis: dose-response relationship of selective serotonin reuptake inhibitors in major depressive disorder. Am J Psychiatry. 2016;173(2):174-183. 
2. Coleman JA, Green EM, Gouaux E. X-ray structures and mechanism of the human serotonin transporter. Nature. 2016;532(7599):334-339.

3. Wohleb ES, Franklin T, Iwata M, Duman RS. Integrating neuroimmune systems in the neurobiology of depression. Nat Rev Neurosci. 2016;17(8): 497-511.

4. Janssen DG, Caniato RN, Verster JC, Baune BT. A psychoneuroimmunological review on cytokines involved in antidepressant treatment response. Hum Psychopharmacol. 2010;25(3):201-215.

5. Goldsmoth DR, Papaport MH, Miller BI. A meta-analysis of blood cytokine network alterations in psychiatric patients: comparisons between schizophrenia, bipolar disorder and depression. Mol Psychiatry. 2016;21(12):1696-1709.

6. Dowlati Y, Herrmann N, Swardfager W, et al. A meta-analysis of cytokines in major depression. Biol Psychiatry. 2010;67(5):446-457.

7. Hannestad J, DellaGioia N, Bloch M. The effect of antidepressant medication treatment on serum levels of inflammatory cytokines: a meta-analysis. Neuropsychopharmacology. 2011;36(12): 2452-2459.

8. Yoshimura R, Hori H, Ikenouchi-Sugita A, et al. Plasma levels of interleukin-6 and selective serotonin reuptake inhibitor response in patients with major depressive disorder. Hum Psychopharmacol. 2013;28(5): 466-470.

9. Yoshimura R, Umene-Nakano W, Hoshuyama T, et al. Plasma levels of brain-derived neurotrophic factor and interleukin-6 in patients with dysthymic disorder: comparison with age- and sex-matched major depressed patients and healthy controls. Hum Psychopharmacol. 2010;25(7-8): 566-569.

10. Aniszewska A, Chłodzińska N, Bartkowska K, Winnicka MM, Turlejski K, Djavadian RL. The expression of interleukin-6 and its receptor in various brain regions and their roles in exploratory behavior and stress responses. J Neuroimmunol. 2015;284:1-9.

11. Maes M. Evidence for an immune response in major depression: a review andhypothesis. Prog Neuropsychopharmacol Biol Psychiatry. 1995;19(1):11-38

12. Hiles SA, Baker AL, de Malmanche T, Attia J. A meta-analysis of differences in IL-6 and IL-10 between people with and without depression: exploring the causes of heterogeneity. Brain Behav Immun. 2012; 26(7):1180-1188.

13. Katoh Y, Uchida S, Kawai M, et al. Onset of clinical and plasma concentration of fluvoxamine in Japanese patients. Biol Pharm Bull. 2010; 33(12):1999-2002.

14. Iron J. Fluvoxamine in the treatment of anxiety disorders. Neuropsychiatr Dis Treat. 2005;1(4):289-299.
15. Omori IM, Watanabe N, Nakagawa A, et al; Meta-analysis of new generation antidepressants (MANGA) study group. Efficacy, tolerability and side-effect profile on fluvoxamine for major depression: metaanalysis. J Psychopharmacol. 2009;23(5):539-550.

16. Hamilton M. Rating scale for depression. JNeurol Neurosurg Psychiatry. 1960;23:56-62.

17. Hori H, Yoshimura R, Ueda N, Ikenouchi-Sugita A, Umene-Nakano W, Nakamura J. A case with occurring adverse effects when cross-over titration from fluvoxamine to paroxetine associated with increasing the plasma fluvoxamine level in major depressive disorder. World J Biol Psychiatry. 2009;10(4 Pt 2):620-622.

18. O’Brien SM, Scully P, Fizgerald P, Scott LV, Dinan T. Plasma cytokine profiles in depressed patients who fail to respond to selective serotonin reuptake inhibitor therapy. J Psychiatry Res. 2007;41(3-4):326-331.

19. Mastorakos G, Weber JS, Magiakou GA, Gunn H, Chrousos GP. Hypothalamic-pituitary-adrenal axis activation and stimulation of systemic vaspressin secretion by recombinant interleukin- 6 in humans: potential implications for the syndrome of inappropriate vasopressin secretion. J Clin Endocrinol Metab. 1994;79(4):934-939.

20. Chrousos GP. The hypothalamic-pituitary-adrenal axis and immune mediated inflammation. N Engl J Med. 1995;332(20):1351-1362.

21. Dentino AN, Piper CF, Rao KMK. Association of interleukin-6 and other biological variables with depression in older people living in the community. Am J Geriatr Soc. 1999;47(1):6-11.

22. Hayley S, Poulter MO, Merali Z, Anisman H. The pathogenesis of clinical depression: stressor-and cytokine-induced alteration of neuroplasticity. Neuroscience. 2005;135(3):659-678.

23. Li M, Yao W, Li S, Xi J. Norepinephrine induces the expression of interleukin-6 via $\beta$-adrenoreceptor-NAD(P)H oxidase system-NF- $\mathrm{\kappa B}$ dependent signal pathway in U937 macrophages. Biochem Biophys Res Coтmun. 2015;460(4):1029-1034.

24. Ueda N, Yoshimura R, Shinkai K, Nakamura J. Plasma levels of catecholamine metabolites predict the response to sulpiride or fluvoxamine in major depression. Pharmacopsychiatry. 2002;35(5):175-181.

25. Jimmerson DC, Insel TR, Reus VI, Kopin IJ. Increased plasma MHPG in dexamethasone-resistant depressed patients. Arch Gen Psychiatry. 1983;40(2):173-176.

26. Hanisch UK. Microglia as a source and target of cytokines. Glia. 2002; 40(2):140-155.

27. Banks WA, Kastin AJ, Broadwell RD. Passage of cytokines across the blood-brain barrier. Neuroimmunomudulation. 1985;2(4):241-248.
Neuropsychiatric Disease and Treatment

\section{Publish your work in this journal}

Neuropsychiatric Disease and Treatment is an international, peerreviewed journal of clinical therapeutics and pharmacology focusing on concise rapid reporting of clinical or pre-clinical studies on a range of neuropsychiatric and neurological disorders. This journal is indexed on PubMed Central, the 'PsycINFO' database and CAS,

\section{Dovepress}

and is the official journal of The International Neuropsychiatric Association (INA). The manuscript management system is completely online and includes a very quick and fair peer-review system, which is all easy to use. Visit http://www.dovepress.com/testimonials.php to read real quotes from published authors. 